\title{
Traditional fermented products - a good source for beneficial lactic acid bacteria
}

\section{Editorial}

Modern diets of the western world are very different than the traditional ones which many researchers and consumers are now realizing that the latter are not only nutritious but also can provide benefits beyond their nutritional properties. Almost all current eating regimes of modern societies include the consumption of elevated amounts of processed foods and ready-to-eat products that contain numerous chemicals or refined additives (such as conservants, stabilizers, emulsifiers, salts, carbohydrates) that are used to increase shelf-life, flavour and physical properties. In addition, most people do not follow proper eating guidelines and fast for long periods of time and then overeat afterwards instead of eating several smaller meals throughout the day. All of these unhealthy foods and bad eating habits are important causes of the increase incidence of obesity, high cholesterol levels in blood, high blood pressure, diabetes and many other health problems including certain types of cancers. At the same time traditional fermented food products that contain low levels of preservatives and that can provide health promoting benefits have only recently been re-incorporated as part of normal diets. Traditional fermented foods such as bread, cheese, salami, olives, beer and wine, have been prepared and consumed for thousands of years using locally available materials and more importantly the methods of preparation are strongly linked to culture and tradition; the preparation of these foods still remains a household art in many isolated regions and are normally passed down from generation to generation. These foods have special organoleptic qualities and some even possess health promoting properties due to the presence of a biologically diverse microbiota, especially Lactic Acid Bacteria (LAB) present in the raw material or as a contaminant from the producers or instruments used to prepare them. These microorganisms are important genetic reservoirs and hold great biotechnological and health improving potentials that should be exploited. These beneficial microorganisms are not only involved in modifying the sensorial and technological aspects of foods, mainly through the release of lactic acid and flavour compounds, but many exhibit probiotic properties. Because of the numerous beneficial properties that have been attributed to LAB, these are the most commonly used probiotic microorganisms that have been defined by the World Health organization as live microorganisms which when administered in adequate amounts confer a health benefit on the host. Some of the health claims attributed to probiotics include the improvement of the normal microbiota and stabilization of the gut mucosal barrier, prevention of infectious diseases and food allergies, hypocholesterolemic, anti-mutagenic and anti-carcinogenic properties, immune system modulation, prevention of inflammatory bowel diseases, and alleviation of lactose intolerance. They have also been shown to provide essential compounds such as vitamins, hydrolytic enzymes, bacteriocins, and other bioactive compounds. Now-a-days, most probiotic foods are milk-based, although many fermented cereals, with added probiotic cultures, are now appearing in specialized markets. However, only limited knowledge of LAB content in traditional fermented food products is currently available and very little has been done in the line of searching these products as a natural source of beneficial LAB. Cereals have a high nutritional value (vitamins, proteins, dietary fiber, energy, and minerals) and are
Volume I Issue 4 - 2014

\author{
Svetoslav Dimitrov Todorov,' Jean Guy \\ LeBlanc $^{2}$ \\ 'Department of Veterinary, Federal University of Vicosa, Brazil \\ ${ }^{2}$ CERELA-CONICET, C.P. 400, Tucuman, San Miguel de Tucumán, \\ Argentina
}

Correspondence: Svetoslav Dimitrov Todorov, Department of Veterinary, Federal University of Vicosa, UFV Campus, 36570 900,Viçosa, Minas Gerais, Brazil,Tel 55 II 98239 956।, Emailslavi310570@abv.bg

Received: August 02, 2014 | Published: August 04, 2014

cultivated on more than $73 \%$ of agricultural soil while contributing to more than $60 \%$ of the world's food production. LAB are frequently involved in the fermentation of the cereals and these products have a long history of use in traditional medicine because of their beneficial properties.

Other fermented food products such as salami, cheese, fruits and vegetables can be a good source of beneficial LAB as well. Consumers can appreciate the highly organoleptic and nutritional characteristics of these products and in the same time can be consuming a high number of LAB with beneficial characteristics.

LAB may contribute to the extension of the self life of the products by producing various antimicrobial substances, including bacteriocins, lactic acid, hydrogen peroxide, fatty acids, diacetyl and other low molecular weight compounds during fermentation. In fact LAB can have play beneficial role in the production and conservation of the food products from one side and been beneficial for the consumers as a probiotic on the later stage. It was demonstrated that without even knowing the basic theories of microbiology, ancient populations were able to optimize the biotechnological aspects of fermentation giving rise to foods and beverages that were not only useful because of their nutritive and social aspects, but also provided healthpromoting and curative properties, many of which are just recently being described by concrete scientific research. These foods thus merit further scientific investigations in order to understand exactly which microorganisms could be useful to be used as biotechnological tools for the development of new and improved foods or used as novel fountains of beneficial LAB that could be used in a wide range of probiotic applications

\section{Acknowledgements}

None.

\section{Conflict of interest}

Author declares that there is no conflict of interest. 